\title{
Electroacupuncture counteracts the development of thermal hyperalgesia and the alteration of nerve growth factor and sensory neuromodulators induced by streptozotocin in adult rats
}

\author{
L. Manni • F. Florenzano • L. Aloe
}

Received: 17 November 2010 / Accepted: 17 February 2011 /Published online: 23 March 2011

(C) Springer-Verlag 2011

\begin{abstract}
Aims/hypothesis Diabetes is considered the leading cause of neuropathies in developed countries. Dysfunction of nerve growth factor (NGF) production and/or utilisation may lead to the establishment of diabetic neuropathies. Electroacupuncture has been proved effective in the treatment of human neuropathic pain as well as in modulating NGF production/activity. We aimed at using electroacupuncture to correct the development of thermal hyperalgesia and the tissue alteration of NGF and sensory neuromodulators in a rat model of type 1 diabetes.

Methods Adult rats were injected with streptozotocin to induce diabetes and subsequently treated with lowfrequency electroacupuncture for 3 weeks. Variation in thermal sensitivity was studied during the experimental course. Hindpaw skin and spinal cord protein content of
\end{abstract}

Electronic supplementary material The online version of this article (doi:10.1007/s00125-011-2117-5) contains supplementary material, which is available to authorised users.

L. Manni $\cdot$ F. Florenzano $\cdot$ L. Aloe

Institute of Neurobiology and Molecular Medicine,

National Research Council,

Rome, Italy

F. Florenzano

Confocal Microscopy Unit, National Research Council,

S. Lucia Foundation,

Rome, Italy

Present Address:

L. Manni $(\square)$

CNR-National Research Council of Italy,

Cell Biology and Neurobiology Institute,

via del Fosso di Fiorano 64,

00143 Rome, Italy

e-mail: luigi.manni@inmm.cnr.it
NGF, NGF receptor tyrosine kinase A (TrkA), substance P (SP), transient receptor potential vanilloid 1 (TRPV1) receptor and glutamic acid decarboxylase-67 (GAD-67) were measured after electroacupuncture treatments. The skin and spinal cord cellular distribution of TrkA was analysed to explore NGF signalling.

Results Early after streptozotocin treatment, thermal hyperalgesia developed that was corrected by electroacupuncture. The parallel increases in NGF and TrkA in the spinal cord were counteracted by electroacupuncture. Streptozotocin also induced variation in skin/spinal TrkA phosphorylation, increases in skin SP and spinal TRPV1 and a decrease in spinal GAD-67. These changes were counteracted by electroacupuncture.

Conclusions/interpretation Our results point to the potential of electroacupuncture as a supportive therapy for the treatment of diabetic neuropathies. The efficacy of electroacupuncture might depend on its actions on spinal/peripheral NGF synthesis/utilisation and normalisation of the levels of several sensory neuromodulators.

Keywords Electroacupuncture - GAD-67 · Hyperalgesia . Nerve growth factor · Rats · Spinal cord · Streptozotocin . Substance P. Transient receptor potential vanilloid receptor 1

\begin{tabular}{|c|c|}
\hline Abbreviations & \\
\hline EA & Electroacupuncture \\
\hline GABA & $\gamma$-Aminobutyric acid \\
\hline GAD-67 & Glutamic acid decarboxylase- 67 \\
\hline GAPDH & $\begin{array}{l}\text { Glyceraldehyde-3-phosphate } \\
\text { dehydrogenase }\end{array}$ \\
\hline NGF & Nerve growth factor \\
\hline $\mathrm{p}^{\text {Tyr496}}-$ TrkA & Phosphorylated tyrosine kinase A \\
\hline SP & Substance P \\
\hline
\end{tabular}


STZ

TrkA

TRPV1

\section{Streptozotocin}

Tyrosine kinase A

Transient receptor potential receptor vanilloid 1

\section{Introduction}

Neuropathy is the most common complication of diabetes and the greatest source of morbidity in diabetes patients [1]. Painful neuropathy is associated with behavioural manifestations of sensory dysfunctions, and neurochemical alterations of biological mediators in the peripheral nervous system [2]. In particular, alterations in the content of neurotrophin nerve growth factor (NGF) in tissues (skin, muscle and serum) [3] are associated with spinal variations in substance $\mathrm{P}$ (SP) [3] and transient receptor potential vanilloid 1 (TRPV1) receptor $[4,5]$. These latter are known to be under the control of NGF [6-8] and have been related to diabetes-induced neuropathy.

Diabetic neuropathy could benefit from NGF-based therapies [9], but the clinical use of NGF has been hampered by the development of important side effects, such as hyperalgesia, pain and autonomic dysfunctions [10].

Acupuncture-based therapies in diabetic patients and animal models have been validated, especially in the treatment of painful neuropathies [11]. From the Western perspective, the pain-relieving effects are based on the establishment of central inhibitory mechanisms triggered by the activation of peripheral sensory afferents elicited by needle stimulation [12]. Recent studies have demonstrated that sensory stimulation by electroacupuncture can have regulatory effects on NGF, NGF signalling (reviewed by Manni et al. [13]) and several NGF-regulated molecules, such as SP and TRPV1 [14]. These effects have been demonstrated in the nervous system and other tissues in animal models of metabolic, neurological and inflammatory disorders [15-22]. The efficacy of acupuncture and electroacupuncture in neuropathic pain $[12,23]$ and their actions on the inhibitory neurotransmitter $\gamma$-aminobutyric acid (GABA) [24] have also been reported. Interestingly, GABA has a role in the generation and maintenance of diabetesinduced sensory dysfunctions $[25,26]$ and the activitydependent regulation of spinal NGF synthesis [27].

It has been proposed that aberrant spinal processing of sensory signalling may be involved in generating sensory dysfunctions in diabetes [2]. Among others, the streptozotocin-induced type-1 diabetes model in rodents is characterised by early functional abnormalities, including thermal hyperalgesia $[5,28]$, associated with spinal deregulation of TRPV1 [5, 29], SP [3] and GABA [25, 26].

In the present study we investigated how electroacupuncture could affect thermal hyperalgesia and modulate NGF content and activity at an early stage after diabetes induction with streptozotocin in rats [30]. Our analysis was done by measuring NGF, tyrosine kinase A (TrkA) and its phosphorylated form, SP, TRPV1 and GABA protein contents in the hindpaw skin and spinal cord, these two tissues being sites of the peripheral and central actions of electroacupuncture in modulating neuropathic pain $[23,24$, 31]. The tissue distribution of TrkA was also analysed to further explore the structures involved in NGF signalling.

\section{Methods}

Animals Adult female Sprague-Dawley rats (HarlanNossan, Correzana, Italy) weighing 200-220 g were housed for 1 week under constant conditions $\left(21 \pm 1^{\circ} \mathrm{C}\right.$, light on from 7:00 to 19:00 hours). Food and water were freely available throughout the experiment. Animal care procedures were conducted in conformity with Italian and international laws and policies (EEC Council Directive 86/609, OJ L 358,1, 12 December 1987; NIH Guide for the Care and Use of Laboratory Animals, NIH Publication No. 85-23, 1985). All experiments were performed between 9:00 and 12:00 hours.

Experimental design Streptozotocin is selectively toxic to the insulin-producing cells of the pancreas and has been widely used to produce animal models of type 1 diabetes [30]. Our rats were treated with a single i.p. injection of $65 \mathrm{mg} / \mathrm{kg}$ streptozotocin (Catalogue No. S0130, SigmaAldrich, Milan, Italy) dissolved in citrate buffer, $\mathrm{pH} 4.5$ [30]. One week after streptozotocin treatment, the establishment of diabetes was checked with an Accutrend GC (Roche Diagnostic, Mannheim, Germany) glucose analyser. Rats with blood glucose levels above $16.65 \mathrm{mmol} / 1$ were allocated to the streptozotocin experimental groups.

Thirty-six rats were divided into groups as follows: 12 control rats were injected once i.p. with $20 \mathrm{mmol} / \mathrm{l}$ citrate, $\mathrm{pH} 4.5 ; 12$ rats were injected with streptozotocin as described above (STZ group); and 12 rats were injected with streptozotocin and treated twice a week with lowfrequency electroacupuncture (described below) for three consecutive weeks starting 1 week after diabetes induction (STZ+EA group). Control of electroacupuncture by minimal or superficial sensory stimulation (sham electroacupuncture) in diabetic rats was omitted since it does not represent an inert intervention, often producing significant responses comparable to those elicited by acupuncture itself [32]. Electoacupuncture in control rats was also omitted because of its demonstrated different effects in healthy and diseased persons [32]. 
Four weeks after streptozotocin treatment and 1 day after the last electroacupuncture session, ten rats in each group were killed by decapitation. Samples of hindpaw skin and lumbar spinal cord were collected, quickly frozen and stored at $-80^{\circ} \mathrm{C}$ until processed for biochemical assays. Two rats in each group were anaesthetised with $400 \mathrm{mg} / \mathrm{kg}$ of chloral hydrate and transcardially perfused with $4 \%$ paraformaldehyde in PBS; tissue was then removed and processed for immunohistochemistry.

Electroacupuncture Rats in the STZ+EA group received 30 min of electroacupuncture stimulation twice a week for 3 weeks as described by Stener-Victorin et al. [16] and Manni et al. [17]. During each electroacupuncture treatment, rats were sedated with an i.p. injection of chloral hydrate $(200 \mathrm{mg} / \mathrm{kg})$. Stimulation was applied bilaterally at the traditional acupoint Zusanli (on the anterior lateral side of the leg close to the anterior crest of the tibia). Such stimulation has been proved effective for the treatment of hyperalgesia as well as neuropathic and inflammatory pain [33]. The needles (Hegu, Landsbro, Sweden) were inserted at the same point bilaterally to a depth of $0.3-0.5 \mathrm{~cm}$ and then attached by clip electrodes to an electrical stimulator (Acus II; Cefar, Malmö, Sweden). The points were stimulated with a low burst frequency of $2 \mathrm{~Hz}$; each pulse was a square wave with duration $0.18 \mathrm{~ms}$, a burst length $0.1 \mathrm{~s}$, and internal burst frequency of $80 \mathrm{~Hz}$. The intensity (1.0-1.5 mA) was monitored by checking for local muscle contractions to reflect the activation of muscle-nerve afferents. The location and type of stimulation were the same in all rats. Rats in the control and STZ groups were manipulated and sedated in a manner similar to those in the STZ+EA group.

Hotplate test Thermal nociception was measured before streptozotocin injection, on day 8 after streptozotocin injection (just before the start of the electroacupuncture treatment protocol) and the day after the last electroacupuncture session, immediately before the animals were killed. A hotplate apparatus (Socrel Hot-Plate model DS37; Ugo Basile, Comerio, Italy) was used as described by Aloe and Manni [14]. The temperature was set at $50 \pm 0.2^{\circ} \mathrm{C}$ and the cutoff time was $60 \mathrm{~s}$. Pain sensitivity was assessed with a digital stopwatch by measuring latency to the first episode of hindpaw withdrawal and/or licking.

NGF assay Tissue content of NGF in each group was assessed using a commercial ELISA (DY556; R\&D Systems, Minneapolis, MN, USA), following the instructions of the manufacturer. Tissue samples were homogenised by ultrasonication in extraction buffer (Tris-acetate $20 \mathrm{mmol} / \mathrm{l}, \mathrm{pH} 7.5, \mathrm{NaCl} 150 \mathrm{mmol} / \mathrm{l}$, EDTA $1 \mathrm{mmol} / \mathrm{l}$, EGTA $1 \mathrm{mmol} / \mathrm{l}$, sodium pyrophosphate $2.5 \mathrm{mmol} / \mathrm{l}$, orthovanadate $1 \mathrm{mmol} / \mathrm{l}, \mathrm{NaF} 100 \mathrm{mmol} / \mathrm{l}$, phenylmethylsulphonyl fluoride $1 \mathrm{mmol} / \mathrm{l}$, leupeptin $1 \mu \mathrm{g} / \mathrm{ml}$ ) and centrifuged at $4{ }^{\circ} \mathrm{C}$ for $10 \mathrm{~min}, 10,000 \times g$, and supernatant fractions were recovered. The protein concentrations in tissue sample extracts were determined with the Bio-Rad DC Protein Assay (500-0116; Life Science Group, Milan, Italy). The amount of NGF was determined from the regression line for the NGF standard (ranging from 15.6 to $1,000 \mathrm{pg} / \mathrm{ml}$ of rat $\mathrm{NGF}$ ).

SP assay Tissue samples were homogenised by ultrasonication in $2 \mathrm{~mol} / 1$ acetic acid at $4^{\circ} \mathrm{C}$. After centrifugation at $8,000 \times g$ for $20 \mathrm{~min}$, the supernatant fractions were collected, dried and resuspended in deionised water, and the protein concentrations were determined with the Bio-Rad DC Protein Assay. The content of SP in tissues was assayed with the Substance P EIA Kit (Cayman Chemical, Ann Arbor, MI, USA), following the instructions of the manufacturer.

Western blotting Sample extracts used for NGF assay were also used for western blotting and analysis of tissue levels of TrkA, phosphorylated TrkA ( p $^{\text {Tyr496}}-$ TrkA), TRPV1, the GABA-synthesising enzyme glutamic acid decarboxylase67 (GAD-67) and glyceraldehyde-3-phosphate dehydrogenase (GAPDH). Samples $(20 \mu \mathrm{g}$ total protein) were dissolved with loading buffer $(0.1 \mathrm{~mol} / \mathrm{l}$ Tris- $\mathrm{HCl}$ buffer, pH 6.8, containing $0.2 \mathrm{~mol} / \mathrm{l}$ DTT, $4 \%$ SDS, $20 \%$ glycerol, and $0.1 \%$ bromophenol blue), separated by SDS-PAGE, and electrophoretically transferred to polyvinylidene fluoride (PVDF) membranes. The membranes were incubated for $1 \mathrm{~h}$ at room temperature with $5 \%$ non-fat dry milk dissolved in TBST (10 mmol/l Tris, $\mathrm{pH} 7.5,100 \mathrm{mmol} / \mathrm{l} \mathrm{NaCl}$ and $0.1 \%$ Tween-20). Membranes were washed three times for $10 \mathrm{~min}$ each in TBST and then incubated overnight at $4{ }^{\circ} \mathrm{C}$ with rabbit anti-TrkA (sc118), mouse anti-p ${ }^{\text {Tyr496 }}$-TrkA (sc8058), rabbit anti-TRPV1 (sc28759) or rabbit antiGAPDH (sc25778) (all from Santa Cruz Biotechnology, Santa Cruz, CA, USA) or mouse anti-GAD67 (MAB5406, Millipore, Milan, Italy) as primary antibodies. Membranes were washed three times for $10 \mathrm{~min}$ each in TBST and incubated for $1 \mathrm{~h}$ with either horseradish peroxidaseconjugated anti-rabbit IgG (Cell Signalling Technology, Danvers, MA, USA) or horseradish peroxidase-conjugated anti-mouse IgG (Cell Signalling Technology) as the secondary antibody. The blots were developed with ECL Chemiluminescent HRP Substrate (Millipore) as the chromophore. The public domain Image J software (http://rsb.info.nih.gov/ij/) was used for gel densitometry and protein quantification following the method described at http://lukemiller.org/index. php/2010/11/analyzing-gels-and-western-blots-with-image-j/. The integrated density of GAPDH bands was used as the normalising factor. Statistical evaluation was performed on 
two separate gel runs/blots carried out using three different sets of samples ( $n=6$ for each experimental group).

$\operatorname{TrkA}$ confocal immunofluorescence The distribution of TrkA in hindpaw skin and spinal cord was analysed by immunofluorescence. Coronal sections (thickness $20 \mu \mathrm{m}$ ) from these tissues were preincubated with $10 \%$ normal goat serum in PBS containing $0.1 \%$ Triton X-100 (PBST) for $2 \mathrm{~h}$ and then incubated overnight at $4^{\circ} \mathrm{C}$ with primary antibody against TrkA (sc118; Santa Cruz; 1:100), in PBST+1\% goat serum. To assess the staining specificity, the first antibody was replaced with purified non-specific rabbit IgG. After washing with PBST, the slides were incubated for $1 \mathrm{~h}$ at room temperature with Alexa Fluor 488 goat anti-rabbit IgG (1:250; Invitrogen, Milan, Italy). The last step was two rinses in PBS and 10 min incubation with the Hoechst reagent (1 ng/ml; Invitrogen) for visualisation of nuclei. Sections were then coverslipped and examined under a confocal laser scanning microscope (Leica SP5; Leica Microsystems, Milan, Italy). One confocal setting was used for acquisition of the three groups in skin and another for spinal cord. For the production of figures, the brightness and contrast of images were adjusted by leaving a tissue fluorescence background for visual assessment of the features with the lowest fluorescence intensity and to aid comparison among the different experimental groups. From a qualitative point of view, three levels of fluorescence intensity were identified: light, medium and high. For TrkA-positive fibres, Z-stack procedures were used to acquire several optical sections, which were projected in the final images. Boundaries and subdivisions of the spinal cord structures were identified with reference to the atlas of Paxinos and Watson [34].

Statistical analysis Statistical evaluations were performed using the StatView software (SAS Institute, Cary, NC, USA) for Windows and data were expressed as mean \pm SEM. For the hotplate test, data were analysed by ANOVA for the three sessions as repeated measures. Western blot $(n=6$ for each experimental group), ELISA and enzyme immunoassay ( $n=10$ for each experimental group) analyses were evaluated by one-way ANOVA. Post hoc comparisons were performed using Tukey's honestly significant difference test. A $p$ value less than 0.05 was considered significant.

\section{Results}

A significant elevation of plasma glucose level in experimental rats was found 1 week after a single injection of streptozotocin (STZ group, 22.2 $\pm 0.88 \mathrm{mmol} / \mathrm{l}$; control group, $6.99 \pm 1.16 \mathrm{mmol} / \mathrm{l}, p<0.05)$, confirming the development of diabetes.

During this early stage, STZ group rats displayed a significant reduction in the heat latency time (Fig. 1). Four weeks after diabetes induction, the latency to painful stimulation was still shorter compared with controls. On the other hand, rats in the STZ+EA group presented a latency response to thermal pain which was nonsignificantly longer than that of the controls.

Streptozotocin caused a significant increase in NGF protein content in hindpaw skin (Fig. 2a) compared with control rats. Electroacupuncture in streptozotocin-treated rats did not elicit significant variation in NGF levels in the skin when compared with the STZ group $(p>0.05)$. In the spinal cord (Fig. 2b), the level of NGF was significantly increased by diabetes induction; electroacupuncture treatment of diabetic rats significantly $(p<0.05)$ decreased the NGF level (Fig. 2b).

As shown in Fig. 2c, streptozotocin provoked about twofold elevation of skin SP content when compared with control rats $(p<0.05)$, and electroacupuncture deeply counteracted this effect (STZ vs STZ+EA $p<0.05$ ), decreasing it well below the control level. On the other hand, spinal SP (Fig. 2d) was lowered by streptozotocin $(p<0.05)$ compared with the control. However, electroacupuncture treatment only slightly decreased SP level compared with the STZ group (Fig. 2d).

Densitometry of western blots (Fig. 3a) revealed that the NGF receptor TrkA content in the skin of diabetic animals underwent a non-significant increase (Fig. 3b). However, after streptozotocin treatment, electroacupuncture significantly lowered the TrkA level compared with the STZ group. The skin content of $p^{\text {Tyr496}}-$ TrkA (Fig. 3c), the phosphorylated and activated form of TrkA, was significantly increased in diabetic animals compared with controls,

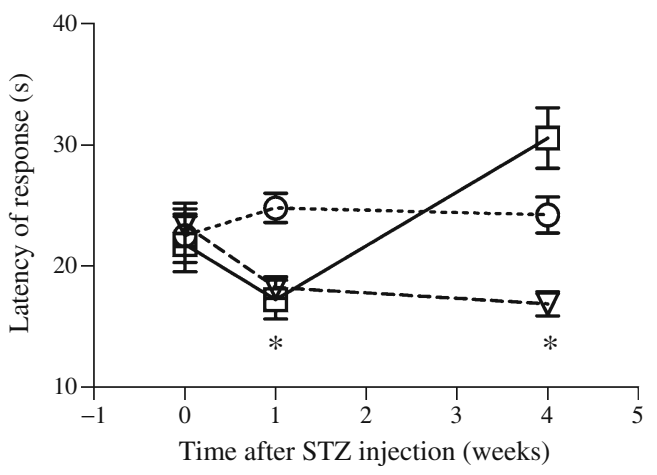

Fig. 1 Hyperalgesia in diabetic rats was normalised by electroacupuncture. Latency of response to thermal pain, as measured by the hotplate test. Triangles, STZ group; squares, STZ+EA group; circles, control group (citrate injection only). Data are mean \pm SEM (repeated measures ANOVA). $n=10$ for each experimental group. ${ }^{*} p<0.05$ vs control group 

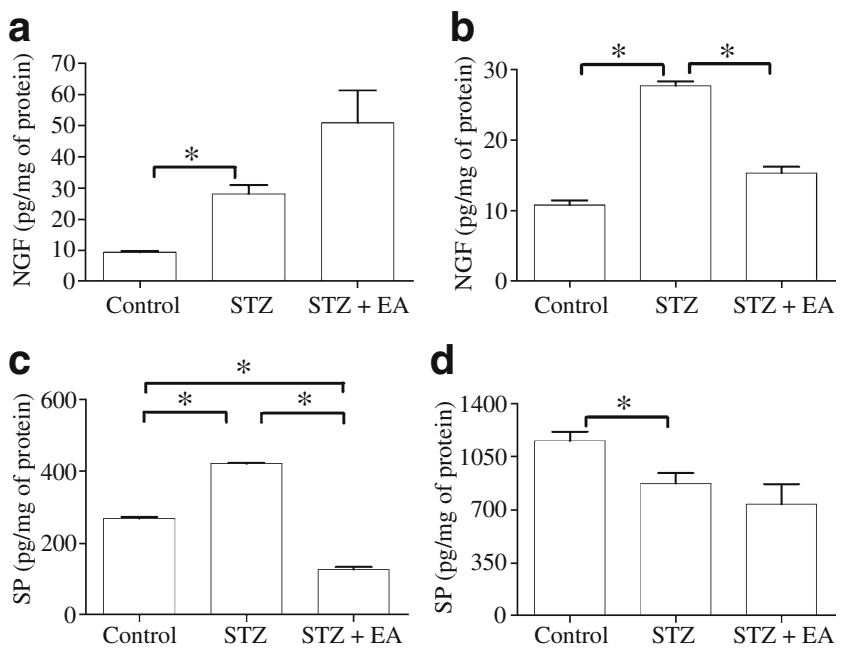

Fig. 2 Effects of electroacupuncture (EA) on NGF and SP in skin and spinal tissues of adult diabetic rats determined 4 weeks after injection of streptozotocin (STZ) with or without EA for 3 weeks starting 1 week after STZ injection. Controls received only a citrate injection, without EA. a NGF in hindpaw skin. b NGF in lumbar spinal cord. c SP in hindpaw skin. d SP in lumbar spinal. Data from the NGF ELISA are mean $\pm \mathrm{SEM}, n=10$ for each experimental group. Data from the SP enzyme immunoassay test are mean \pm SEM, $n=10$ for each experimental group. ${ }^{*} p<0.05$

indicating augmented NGF signalling in streptozotocintreated rats. Electroacupuncture induced a reduction of $\mathrm{p}^{\text {Tyr496}}$-TrkA content in diabetic animals (STZ vs STZ+EA, $p<0.05)$. Streptozotocin also caused an increase in TRPV1
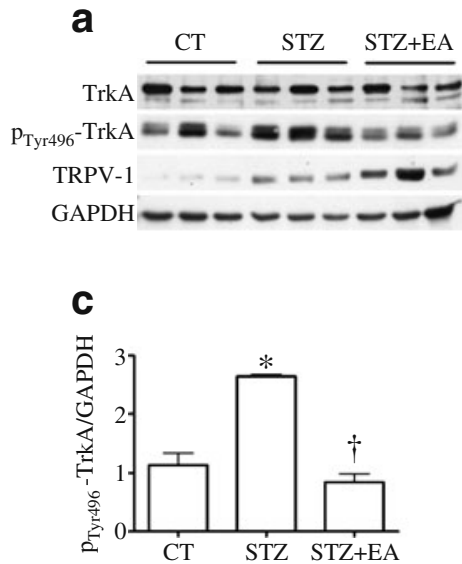

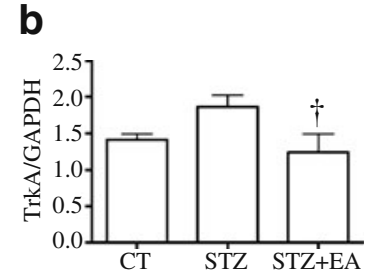

d

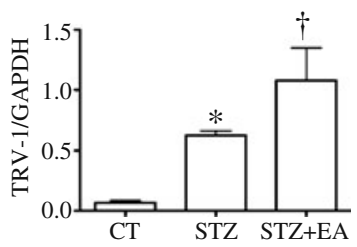

Fig. 3 Electroacupuncture (EA), given for 3 weeks starting 1 week after streptozotocin (STZ) injection, modulated NGF receptor activation and TRPV1 in hindpaw skin of STZ-induced diabetic rats. Controls received only a citrate injection, without EA. a Representative western blots of three samples for each experimental group obtained 4 weeks after STZ injection. b TrkA. c $\mathrm{p}_{\text {Tyr } 496}$-TrkA (activated form of the receptor TrkA). d TRPV1. Data in $b-d$ are ratios of integrated optical densities of TrkA, $\mathrm{p}_{\text {Tyr } 496^{-T r k A} \text { or TRPV1 }}$ to GAPDH bands in gel densitometry analysis, and are mean \pm SEM $(n=6) .{ }^{*} p<0.05$ vs control group; ${ }^{\dagger} p<0.05$ vs STZ group in the skin of diabetic rats (Fig. 3d; control vs STZ group, $p<0.05$ ). Electroacupuncture treatment further increased the TRPV1 level in the skin compared with the STZ group $(p<0.05)$.

TrkA immunofluorescence in hindpaw epidermis did not show qualitative variations in the intensity and distribution pattern among the experimental groups (Fig. 4). In all groups, TrkA immunoreactivity was observed as small vesicles distributed homogeneously (Fig. 4 and electronic supplementary material [ESM] Fig. 1). This TrkA immunoreactivity appeared as tissue background of medium intensity specifically located in the epidermal cell layers I and II (Fig. 4 and ESM Fig. 1). In the basal epidermal layer, small vesicles highly immunoreactive for TrkA filled the cytoplasm of cell groups that alternated with other cell groups which displayed less immunoreactivity (Fig. 4, layer I). In the dermis several small fibres were intensely TrkA immunoreactive. These fibres displayed a tortuous route and were difficult to follow throughout the dermis and epidermis (ESM Fig. 2).

As shown in Fig. 5a, b, streptozotocin did not significantly increase spinal TrkA levels as detected by western blotting (control vs STZ group, $p>0.05$ ), while electroacupuncture decreased spinal TrkA levels in diabetic rats (STZ vs STZ+EA, $p<0.05$ ). The level of $\mathrm{p}^{\text {Tyr496}}-$ TrkA in the spinal cord (Fig. 5a, c) was not significantly increased
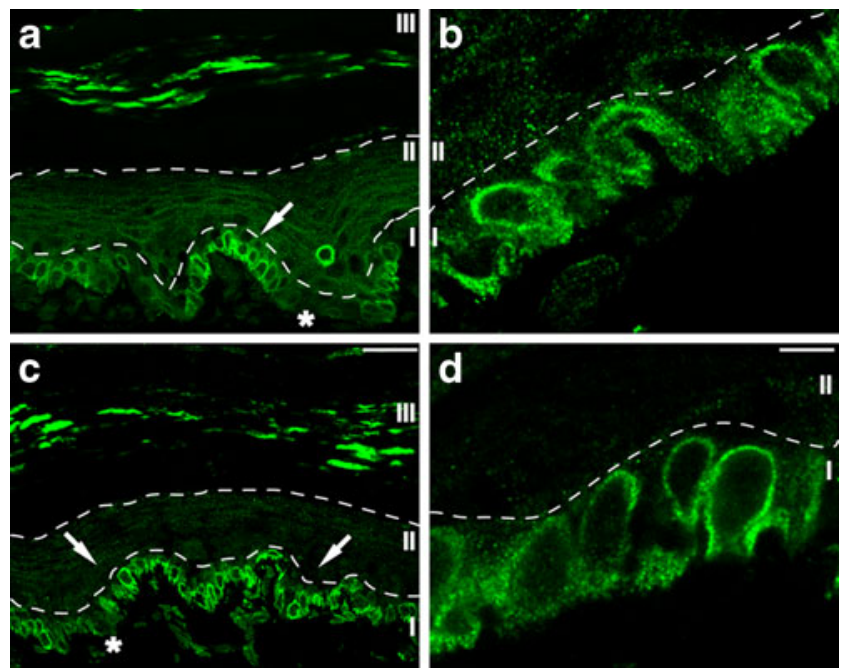

Fig. 4 Confocal images of TrkA immunofluorescence in the epidermis of the STZ and STZ+EA groups. a, b STZ group. c, d STZ+EA group. TrkA was generally produced in the granularsquamous (II) and basal epidermal cell (I) layers as a mediumintensity tissue background. In the basal epidermal layer (I), above the TrkA medium-intensity tissue background, intense TrkA immunoreactive vesicles filled the cytoplasm of cell groups (arrows) as a perinuclear rim leaving the nucleus unstained (see high magnifications in $\mathbf{b}$ and $\mathbf{d}$ ). These cell groups alternated with other cell groups displaying a medium-intensity immunofluorescence background (asterisks). Scale bar: a, c, $50 \mu \mathrm{m} ; \mathbf{b}, \mathbf{d}, 10 \mu \mathrm{m}$ 


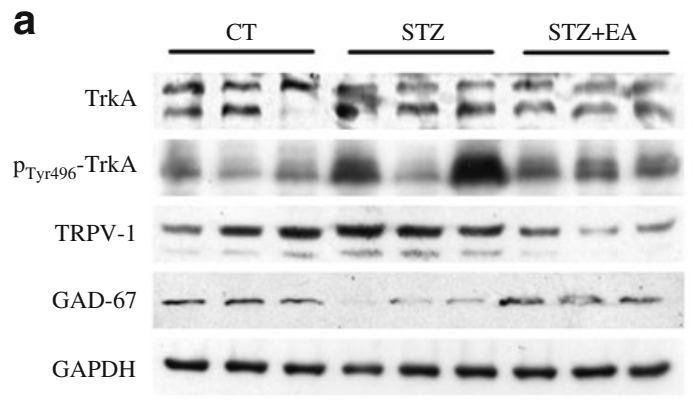

C

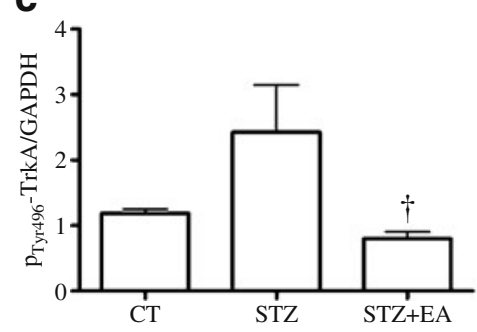

d

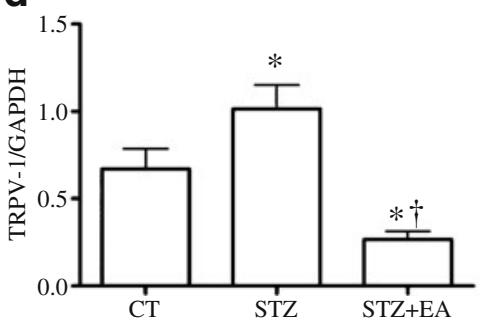

b

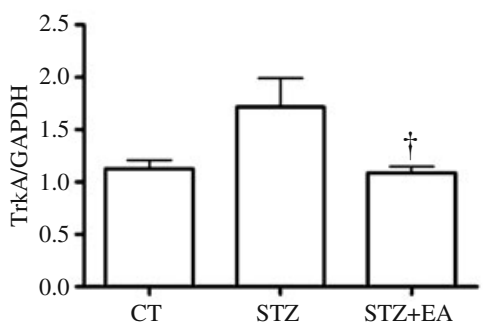

e

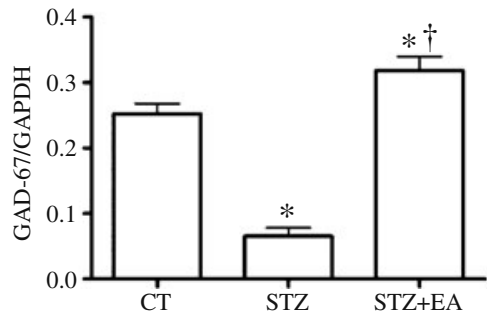

Fig. 5 Electroacupuncture (EA) given for 3 weeks starting 1 week after streptozotocin (STZ) injection, modulated TrkA activation as well as TRPV1 and GAD-67 in lumbar spinal cord of diabetic rats. Controls received only a citrate injection, without EA. a Representative western blots of three samples for each experimental group

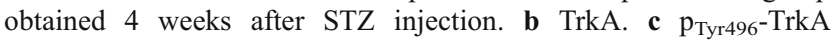

by streptozotocin (control vs STZ group, $p>0.05$ ), but was reduced by electroacupuncture in diabetic animals (STZ vs STZ+EA, $p<0.05)$. The spinal cord level of TRPV1 (Fig. 5a, d) was increased in the STZ group (control vs STZ, $p<0.05$ ), while electroacupuncture counteracted the streptozotocin effect, lowering spinal cord TRPV1 content (control vs STZ+EA and STZ vs STZ+EA, both $p<0.05$ ).

It is known that GABA regulates the abnormal spinal sensory processing in diabetic animals $[25,26]$ and that electroacupuncture exerts actions on GABA [12]. To identify GABA, we analysed the GABA-synthesising enzyme GAD-67. As shown in Fig. 5a, e, GAD-67 protein was downregulated by streptozotocin in the spinal cord (control vs STZ group, $p<0.05$ ), suggesting decreased GABA availability in diabetic rats. Electroacupuncture restored the GAD-67 protein content in the spinal cord of STZ group rats to levels similar to those in the control group (STZ vs STZ+EA, $p<0.05$ ).

TrkA immunofluorescence in the spinal cord showed variations in the distribution pattern and immunoreactive intensity after electroacupuncture in streptozotocin-treated rats (Fig. 6). In all three groups, TrkA immunoreactivity was confined to the dorsal horn structures. In the STZ and control groups, two distribution patterns were recognisable. The first pattern represented the entrance of TrkA positive fibres, which displayed intense immunoreactivity, densely distributed in the mediolateral domains of the white matter above the dorsal horn (dorsolateral fasciculus; Fig. 6, layer w). (activated form of TrkA receptor). d TRPV1. e GAD-67 (GABA biosynthetic enzyme). Data in $\mathbf{b}-\mathbf{e}$ are ratios of integrated optical densities of TrkA, $\mathrm{p}_{\text {Tyr496 }}$-TrkA, TRPV1 or GAD-67 to GAPDH bands in gel densitometry analysis, and are mean $\pm \operatorname{SEM}(n=6) . * p<0.05$ vs control group; ${ }^{\dagger} p<0.05$ vs STZ group
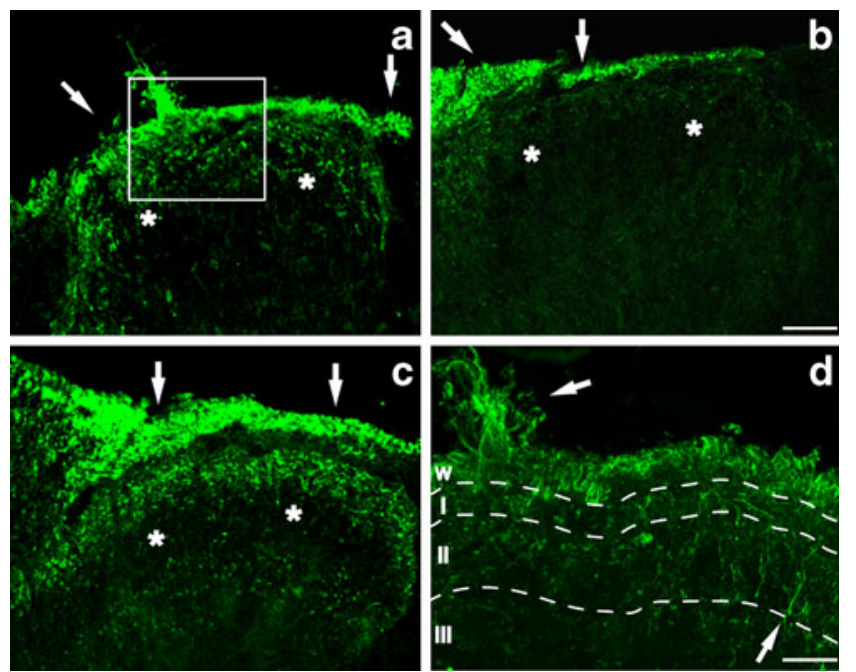

Fig. 6 Confocal images of TrkA immunofluorescence in the dorsal horn of the spinal cord of control, STZ and STZ+EA groups. a, d Control group. b STZ+EA group. c STZ group. TrkA immunofluorescence is present in the white matter of the dorsolateral fasciculus (arrows in $\mathbf{a}, \mathbf{b}$ and $\mathbf{c}$; layer $\mathbf{w}$ in $\mathbf{d}$ ) as bundles of incoming fibres and in the grey matter of the superficial dorsal horn (asterisks in a-c; I, II, III in d) as fibres (arrows in d) and terminals. Note the decrease in TrkA-immunoreactive puncta and terminals in the superficial dorsal horn after electroacupuncture treatment (b, asterisks). Scale bars: a-c $50 \mu \mathrm{m} ; \mathbf{d} 25 \mu \mathrm{m}$ 
The second pattern was observed in the superficial laminae of the dorsal horn, where TrkA-immunoreactive puncta and fibre terminals appeared sparsely and homogeneously distributed in the neuropile (Fig. 6, layers I-III, and ESM Fig. 3). However, in the STZ+EA group the distribution pattern of TrkA immunoreactivity in the superficial laminae of the dorsal horn appeared substantially decreased (Fig. 6c). TrkA-immunoreactive puncta and fibre terminals had disappeared, leaving a tissue background of light intensity. In the white matter, TrkA fibre immunoreactivity in the dorsolateral fasciculus appeared unchanged or slightly decreased in the STZ and control groups.

\section{Discussion}

In the present study we tested the effects of electroacupuncture on the variation in thermal nociceptive threshold and on the contents of NGF, TrkA, $\mathrm{p}^{\text {Tyr496}}$-TrkA and several sensory neuromodulators in paw skin and spinal cord during the early period after diabetes induction by streptozotocin in adult rats. In addition, the distribution of NGF receptor, TrkA, in the hindpaw skin and spinal cord was investigated. Our results demonstrate that electroacupuncture normalises thermal hyperalgesia and affects the spinal regulation of NGF, TrkA, $\mathrm{p}^{\text {Tyr496 }}$-TrkA, SP, TRPV1 and the GABAergic system during the development of type 1 diabetes in rats.

Behavioural and physiological studies have revealed indices of sensory dysfunction in animal models of diabetes [2] that have been associated with dysregulation of NGF synthesis and/or utilisation by NGF-producing tissues $[2,3$, 9]. The streptozotocin-induced diabetes model exhibits two distinct phases, characterised by different sensory abnormalities: an early phase, lasting no less than 4-7 weeks, showing thermal hyperalgesia $[28,35]$, and a late phase displaying thermal hypoalgesia, mechanical allodynia and hyperalgesia [2]. Our data refer to the early presence of thermal hyperalgesia and show that it is associated with an increased NGF protein content in paw skin and spinal cord. We show clearly that streptozotocin induced deregulation of the spinal/skin NGF signalling, which could be responsible at least for the early development of thermal hyperalgesia in diabetic rats and could be corrected by electroacupuncture.

Most reports on the streptozotocin model indicate the presence of sensory abnormalities characterised by late thermal hypoalgesia and mechanical allodynia, similar to the abnormalities seen in human diabetic neuropathy [2]. A parallel decrease in NGF content has been reported in neuronal target tissues of the hindlimb and sciatic nerve of diabetic rats [3]. From the work of our group (L. Manni and L. Aloe, unpublished results) we had an indication of an early increase in NGF induced by streptozotocin in the pancreas, hypothalamus and paw skin (an observation confirmed by the present data), followed by a decline (after 4-6 weeks) below control levels, suggesting differential regulation of NGF content associated with the two phases. It is possible that the presence/action of neuromodulators acting at different sites in the neural circuitry involved in the generation of neuropathic pain could be different in the late streptozotocin-induced diabetes phase. This suggests the need for a more comprehensive study upon the effects of electroacupuncture, as an NGF-modulating approach, on the late phase of streptozotocin-induced diabetes and at different anatomical levels of the nervous system. Such investigations could also show whether the early increases in NGF observed in the spinal cord and skin could have a role in the generation of late central sensitisation of the neural circuitry involved in pain [2].

Three weeks of electroacupuncture sessions completely abolished streptozotocin-induced thermal hyperalgesia in rats, with a concomitant regulatory action on spinal NGF. It has been reported that the effects of electroacupuncture are correlated to the activation of type A and C sensory nerve fibres [36, 37], whose cell bodies are housed in the dorsal root ganglia, resulting in the establishment of skin, spinal and central responses [33, 36, 38, 39]. Our study was restricted to NGF-producing tissues, while the abovementioned considerations suggest the need for an extension of our analysis to NGF-utilising neurons located in the dorsal root ganglia. This will probably help clarify the different effects of streptozotocin and electroacupuncture that we found on skin and spinal cord. Our data show an association among NGF levels, diabetic neuropathy and electroacupuncture effects, which may indicate an important role of the NGF system in the establishment and maintenance of the painful component of early diabetic neuropathy.

The activation of TrkA by streptozotocin in the skin and spinal cord, as revealed by the increase in $\mathrm{p}^{\text {Tyr496}}-$ TrkA, is in line with the increase in NGF availability in diabetic rats and suggests that increased NGF activity participates in the generation of thermal hyperalgesia. Electroacupuncture normalises $\mathrm{p}^{\text {Tyr496}}-$ TrkA in the paw skin and spinal cord of streptozotocin-treated rats. Thus, as well as NGF tissue levels, NGF signalling seems to be counteracted by electroacupuncture. The lack of effect of electroacupuncture on skin NGF could reflect the difference in mechanism(s) regulating its synthesis in skin and neuronal tissues. Indeed, adrenergic-mediated NGF regulation by electroacupuncture has been proposed for peripheral tissues [13], while our present data and previous reports [27] suggest that GABAmediated central regulation of NGF could be triggered by electroacupuncture.

In our skin samples we did not find differences in the distribution and intensity of TrkA immunoreactivity in the epidermis among the experimental groups. Thus, the TrkA 
content after electroacupuncture treatment, as revealed by western blotting, was not related to the TrkA content in the epidermis, as revealed by confocal microscopy. Other sources of TrkA protein in the skin are the polymodal $\mathrm{C}$ fibres, coming from the dorsal root ganglia and terminating in the dermis, which were not included in our morphological analysis. This latter source of TrkA protein could account for the changes in protein content evidenced by western blotting in our material.

TrkA immunoreactivity was decreased by electroacupuncture in puncta and terminals in the superficial dorsal horn of the spinal cord. The source of TrkA immunoreactivity in the superficial dorsal horn is represented by the terminals of unmyelinated polymodal $\mathrm{C}$ fibres coming from the ganglion and terminating in the upper laminae of the dorsal horn [40]. The incoming fibres did not show substantial variation in TrkA immunoreactivity, indicating that the decrease in immunoreactivity was selectively confined to the synaptic circuitry in the dorsal horn and suggesting that it was directly related to in situ downregulation of TrkA protein and not to a loss of incoming TrkA positive fibres. Moreover, the STZ group did not show differences in TrkA immunoreactivity compared with the control group. Consequently, streptozotocin-induced neuropathy does not provoke loss of fibres or terminals, at least at the time point under examination. Thus, the decrease in TrkA immunoreactivity can be ascribed to a selective effect exerted by electroacupuncture on the TrkA protein production of terminals in the dorsal horn, an anatomical location dedicated to the transmission of pain between the periphery and the central nervous system.

The skin levels of SP and spinal TRPV1 varied according to variation in $\mathrm{p}^{\text {Tyr496}}$-TrkA after streptozotocin alone (STZ group) and streptozotocin followed by electroacupuncture (STZ+EA group). The streptozotocin-induced decrease in spinal SP may be controversial in the light of the increased availability of spinal NGF in our diabetic rats. The proposed mechanism of spinal sensitisation mediated by an increase in the level of neurokinin 1 receptor - the SP receptor-in diabetic neuropathy [2] could explain the decreased spinal level of SP and the concomitant behavioural alteration recorded in our STZ group. Furthermore, the variations in skin TRPV1 induced by streptozotocin (STZ group) and streptozotocin followed by electroacupuncture (STZ+EA group) reflected those observed for NGF, suggesting that a common regulatory mechanism could be active in the skin. Our present data do not allow further speculation on this issue. However, since the spinal production of TRPV1 has been correlated with the development of thermal hyperalgesia in diabetic animals [5, 29], our data underline the possibility that the efficacy of electroacupuncture in the control of spinal sensitisation during the development of neuropathic pain states could be mediated by the modulation of NGF and TRPV1 levels.

Information about the spinal GABAergic system gives some clues about the possible mechanism linking electroacupuncture and spinal NGF in our experimental model. GABA neurons play a major role in the spinal modulation of pain perception [41] and in the streptozotocin-induced development of allodynia and hyperalgesia $[25,26]$. The electroacupuncture-induced modulation of spinal GAD67, reflecting GABA availability, could be linked not only to the amelioration of hyperalgesic symptoms but also to the observed variation in the level of NGF, since an elevation of spinal NGF mRNA and protein following $G_{A B A}$ receptor antagonist treatment has been reported in rats [27].

In conclusion, our data indicate that electroacupuncture is able to counteract streptozotocin-induced early thermal hyperalgesia in adult rats. The mechanisms of action of electroacupuncture may be based on the modulation of NGF signalling and of spinal neurotransmitters (e.g. GABA) and neuromodulators (e.g. TRPV1).

Acknowledgements The authors acknowledge the technical contribution of M. L. Rocco (Institute of Neurobiology and Molecular Medicine, CNR, Rome Italy). The authors also acknowledge the contributions of C. Riviello and A. Graziani (Institute of Neurobiology and Molecular Medicine, CNR, Rome, Italy) for animal management and care.

Duality of interest The authors declare that there is no duality of interest associated with this manuscript.

\section{References}

1. Younger DS, Rosoklija G, Hays AP (1998) Diabetic peripheral neuropathy. Semin Neurol 18:95-104

2. Calcutt NA (2002) Potential mechanisms of neuropathic pain in diabetes. Int Rev Neurobiol 50:205-228

3. Tomlinson DR, Fernyhough P, Diemel LT (1996) Neurotrophins and peripheral neuropathy. Philos Trans R Soc Lond B Biol Sci 351:455-462

4. Song JX, Wang LH, Yao L, Xu C, Wei ZH, Zheng LR (2009) Impaired transient receptor potential vanilloid 1 in streptozotocininduced diabetic hearts. Int J Cardiol 134:290-292

5. Pabbidi RM, Yu SQ, Peng S, Khardori R, Pauza ME, Premkumar LS (2008) Influence of TRPV1 on diabetes-induced alterations in thermal pain sensitivity. Mol Pain 4:9

6. Rush RA, Chie E, Liu D, Tafreshi A, Zettler C, Zhou XF (1997) Neurotrophic factors are required by mature sympathetic neurons for survival, transmission and connectivity. Clin Exp Pharmacol Physiol 24:549-555

7. Bennett DL (2001) Neurotrophic factors: important regulators of nociceptive function. Neuroscientist 7:13-17

8. Zhang X, Huang J, McNaughton PA (2005) NGF rapidly increases membrane expression of TRPV1 heat-gated ion channels. EMBO J 24:4211-4223

9. Apfel SC, Kessler JA (1996) Neurotrophic factors in the treatment of peripheral neuropathy. Ciba Found Symp 196:98-108

10. Apfel SC (2002) Nerve growth factor for the treatment of diabetic neuropathy: what went wrong, what went right, and what does the future hold? Int Rev Neurobiol 50:393-413 
11. Head KA (2006) Peripheral neuropathy: pathogenic mechanisms and alternative therapies. Altern Med Rev 11:294-329

12. Zhao ZQ (2008) Neural mechanism underlying acupuncture analgesia. Prog Neurobiol 85:355-375

13. Manni L, Albanesi M, Guaragna M, Barbaro Paparo S, Aloe L (2010) Neurotrophins and acupuncture. Auton Neurosci 157:9-17

14. Aloe L, Manni L (2009) Low-frequency electro-acupuncture reduces the nociceptive response and the pain mediator enhancement induced by nerve growth factor. Neurosci Lett 449:173-177

15. Stener-Victorin E, Lundeberg T, Waldenstrom U et al (2000) Effects of electro-acupuncture on nerve growth factor and ovarian morphology in rats with experimentally induced polycystic ovaries. Biol Reprod 63:1497-1503

16. Stener-Victorin E, Lundeberg T, Cajander S et al (2003) Steroidinduced polycystic ovaries in rats: effect of electro-acupuncture on concentrations of endothelin-1 and nerve growth factor (NGF), and expression of NGF mRNA in the ovaries, the adrenal glands, and the central nervous system. Reprod Biol Endocrinol 1:33

17. Manni L, Lundeberg T, Holmang A, Aloe L, Stener-Victorin E (2005) Effect of electro-acupuncture on ovarian expression of alpha (1)- and beta (2)-adrenoceptors, and p75 neurotrophin receptors in rats with steroid-induced polycystic ovaries. Reprod Biol Endocrinol 3:21

18. Manneras L, Cajander S, Lonn M, Stener-Victorin E (2009) Acupuncture and exercise restore adipose tissue expression of sympathetic markers and improve ovarian morphology in rats with dihydrotestosterone-induced PCOS. Am J Physiol Regul Integr Comp Physiol 296:R1124-R1131

19. Pagani L, Manni L, Aloe L (2006) Effects of electroacupuncture on retinal nerve growth factor and brain-derived neurotrophic factor expression in a rat model of retinitis pigmentosa. Brain Res 1092:198-206

20. Zhao L, Wang Y, Sun N, Liu X, Li L, Shi J (2007) Electroacupuncture regulates TRPM7 expression through the trkA/PI3K pathway after cerebral ischemia-reperfusion in rats. Life Sci $81: 1211-1222$

21. Wang TH, Wang XY, Li XL, Chen HM, Wu LF (2007) Effect of electroacupuncture on neurotrophin expression in cat spinal cord after partial dorsal rhizotomy. Neurochem Res 32:1415-1422

22. Chae Y, Hong MS, Kim GH et al (2007) Protein array analysis of cytokine levels on the action of acupuncture in carrageenaninduced inflammation. Neurol Res 29(Suppl 1):S55-S58

23. Han JS (2004) Acupuncture and endorphins. Neurosci Lett 361:258-261

24. Park JH, Han JB, Kim SK et al (2010) Spinal GABA receptors mediate the suppressive effect of electroacupuncture on cold allodynia in rats. Brain Res 1322:24-29

25. Jolivalt CG, Lee CA, Ramos KM, Calcutt NA (2008) Allodynia and hyperalgesia in diabetic rats are mediated by GABA and depletion of spinal potassium-chloride co-transporters. Pain 140:48-57
26. Morgado C, Pinto-Ribeiro F, Tavares I (2008) Diabetes affects the expression of GABA and potassium chloride cotransporter in the spinal cord: a study in streptozotocin diabetic rats. Neurosci Lett 438:102-106

27. Heese K, Otten U, Mathivet P, Raiteri M, Marescaux C, Bernasconi R (2000) GABA(B) receptor antagonists elevate both mRNA and protein levels of the neurotrophins nerve growth factor (NGF) and brain-derived neurotrophic factor (BDNF) but not neurotrophin-3 (NT-3) in brain and spinal cord of rats. Neuropharmacology 39:449-462

28. Forman LJ, Estilow S, Lewis M, Vasilenko P (1986) Streptozocin diabetes alters immunoreactive beta-endorphin levels and pain perception after $8 \mathrm{wk}$ in female rats. Diabetes 35:1309-1313

29. Kamei J, Zushida K, Morita K, Sasaki M, Tanaka S (2001) Role of vanilloid VR1 receptor in thermal allodynia and hyperalgesia in diabetic mice. Eur J Pharmacol 422:83-86

30. Ferner RE (1992) Drug-induced diabetes. Baillières Clin Endocrinol Metab 6:849-866

31. Xing GG, Liu FY, Qu XX, Han JS, Wan Y (2007) Long-term synaptic plasticity in the spinal dorsal horn and its modulation by electroacupuncture in rats with neuropathic pain. Exp Neurol 208:323-332

32. Lundeberg T, Lund I, Sing A, Naslund J (2009) Is placebo acupuncture what it is intended to be? Evid Based Complement Alternat Med. doi:10.1093/ecam/nep049

33. Ulett GA, Han S, Han JS (1998) Electroacupuncture: mechanisms and clinical application. Biol Psychiatry 44:129-138

34. Paxinos G, Watson C (1982) The rat brain in stereotaxic coordinates. Academic Press, Sydney

35. Gabra BH, Benrezzak O, Pheng LH et al (2005) Inhibition of type 1 diabetic hyperalgesia in streptozotocin-induced Wistar vs spontaneous gene-prone BB/Worchester rats: efficacy of a selective bradykinin B1 receptor antagonist. J Neuropathol Exp Neurol 64:782-789

36. Andersson S, Lundeberg T (1995) Acupuncture-from empiricism to science: functional background to acupuncture effects in pain and disease. Med Hypotheses 45:271-281

37. Kimura A, Sato A (1997) Somatic regulation of autonomic functions in anesthetized animals - neural mechanisms of physical therapy including acupuncture. Jpn J Vet Res 45:137-145

38. Hole K, Berge OG (1981) Regulation of pain sensitivity in the central nervous system. Cephalalgia 1:51-59

39. Kaptchuk TJ (2002) Acupuncture: theory, efficacy, and practice. Ann Intern Med 136:374-383

40. Chung EK, Zhang XJ, Xu HX, Sung JJ, Bian ZX (2007) Visceral hyperalgesia induced by neonatal maternal separation is associated with nerve growth factor-mediated central neuronal plasticity in rat spinal cord. Neuroscience 149:685-695

41. Dickenson AH, Chapman V, Green GM (1997) The pharmacology of excitatory and inhibitory amino acid-mediated events in the transmission and modulation of pain in the spinal cord. Gen Pharmacol 28:633-638 